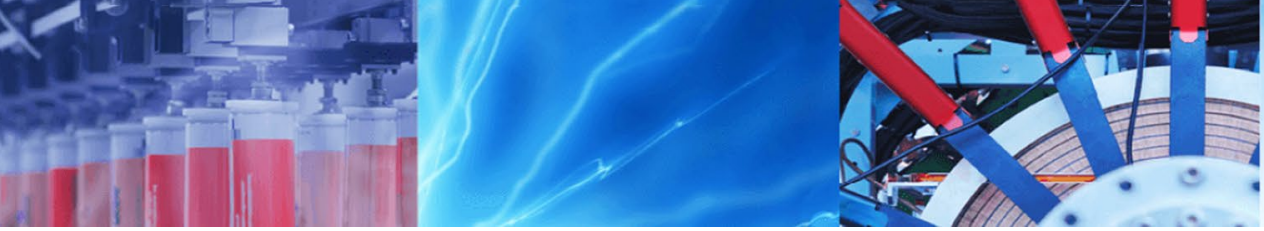

Research Article

\title{
Rolling resistance prediction of off-road tire using advanced simulation and analytical techniques
}

\author{
Zeinab El-Sayegh $^{1}$ (D) Moustafa El-Gindy ${ }^{1}$
}

Received: 3 February 2020 / Accepted: 27 August 2020 / Published online: 2 September 2020

(c) Springer Nature Switzerland AG 2020

\begin{abstract}
This paper focuses on developing a relationship between the rolling resistance coefficient of an off-road truck tire running over different terrains and at various operating conditions. The various operating conditions include tire speed, vertical load, and inflation pressure. The different terrains include dry and moist sand, flooded surface, snow, dense sand, clayey soil, and sandy loam with moisture. The off-road truck tire size 315/80R22.5 is modeled and validated using the finite element analysis technique, while the terrains are modeled and calibrated using smoothed-particle hydrodynamics technique. Artificial neural network and genetic algorithm (GA) are then used to develop a relationship between the rolling resistance coefficient and the terrains and operating conditions. The results of both algorithms are compared to the simulation results in terms of R-square goodness of fit and the mean squared error. Finally, a numerical equation is presented that determines the rolling resistance coefficient as a function of the terrains parameters and the operating conditions. It was found that both techniques provide a suitable solution, however, the GA provides an explicit equation.
\end{abstract}

Keywords Tire modeling · Terrain modeling · Tire-terrain interaction · Rolling resistance $\cdot$ Artificial neural network · Genetic algorithm

\section{Introduction}

The optimization process has been applied to the field of tire mechanics since 1973 [1]. The genetic algorithm (GA) was first introduced by John Holland [2] in 1970s to duplicate the nature actions. Holland was anxious about algorithms that employ binary digits. The "chromosome" used in the GA is an artificial one that has a number of "genes" and each one is represented using binary numbers of 0 or 1. It was noticed that nature has the capability to learn and adapt arbitrary, in other words the nature finds and selects good chromosomes. The GA algorithm is designed to duplicate the nature ability, it employees two mechanisms to solve problems, the encoding and evaluation. The GA relies on reproduction, crossover, selection and mutation which are duplicated by mathematical models. A measure of fitness is implemented for each individual chromosome to accomplish reproduction. During the reproduction phase, the crossover operator is implemented to exchange elements of two chromosomes. Later, the gene value in some of the chromosomes that are randomly chosen are adjusted using the mutation operator.

In 2000, Licheng [3] developed a novel GA algorithm based on immunity, Licheng utilized the local information in order to avoid repetitive work, it was concluded that the novel algorithm is effective and improves the searching ability. Later in 2004, Abe [4] used the GA to optimize the construction of the tire reinforcement. Abe indicated that the if one characteristic is enhanced the quality of another character is affected by sacrificing it. Abe concluded that using the GA the cornering stiffness of the tire increases

Zeinab El-Sayegh, Zeinab.El-Sayegh@uoit.ca; Moustafa El-Gindy, Moustafa.El-Gindy@uoit.ca|'Ontario Tech University, 2000 Simcoe Street North, Oshawa L1H 7K4, Canada. 
more than $15 \%$ in comparison with an indoor drum test performed in control structured environment.

In 2008, Matuvsko [5] implemented the artificial neural network (ANN) to estimate the tire-road frictional forces. Matuvsko added the ANN to the estimator in an attempt to model friction effect, it was concluded that the estimation of the forces was very fast and the system was able to compensate for the change of the model parameters.

In 2012, Cho et al. [6] modified the stiffness and shape of the sidewalls of a run-flat tire in an attempt to investigate the durability and riding comfort. Cho developed a genetic algorithm to replace the CPU time consuming simulations. Cho emphasized on the idea of the run-flat tires and the importance of protecting the sidewall when the pressure level becomes low and verified his proposed method successfully seeks Pareto solutions. In the same year, Cirovic [7] used the Recurrent Neural Network to design a braking torque controller. The primary objective of his research was to model the dynamic change in the braking torque in order to develop a dynamic model able to capture the braking cycle. Cirovic concluded that the braking torque could be dynamically modeled to predict the braking torque under different conditions including applied pressure, the speed and the interface temperature during a braking cycle.

In 2017, Zhu et al. [8] designed a smart tire to estimate the tire forces and the influence of specific factors. The Neural Network algorithm was applied to determine the tire forces and an FTire model was used. It was concluded that the proposed method is efficient and simple.

ANN is an interconnected systems of simple processing units. The processing units are referred to as neurons and they work in parallel to achieve a required result. In most applications the ANN is regarded to as black box that has an input and output. Figure 1 shows the structure of an ANN which is based on the original model developed in 1943 by Mcculloch [9]. The model is an attempt to model the signal processing characteristics of the biological nerve cell in a mathematical approach. It is noted that $w_{i}$

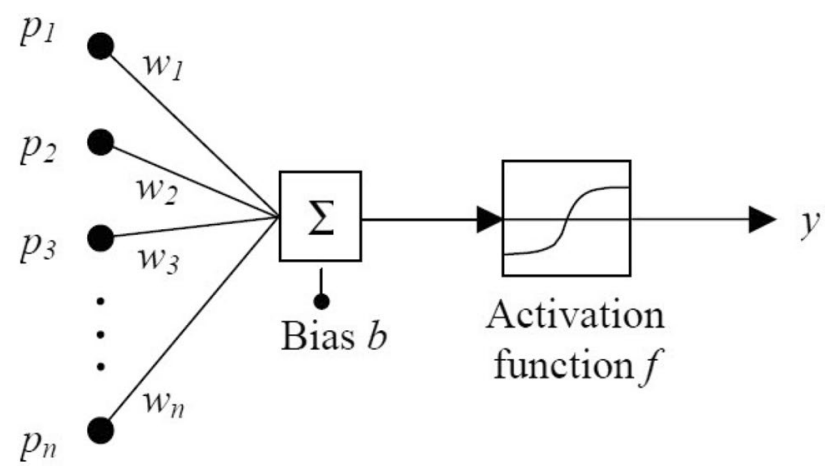

Fig. 1 A model presenting the artificial neuron [9] is the weight of each input channel, $p_{i}, y$ is the output, and $n$ is the number of neurons. The weight of each channel increases or decreases the incoming signal.

The purpose of this paper is to utilize both the ANN and GA algorithms to numerically model a relationship between the rolling resistance coefficient and tire-terrain operating conditions. The tire-terrain operating conditions include tire speed, vertical load, inflation pressure, in addition to the terrain cohesion, angle of shear resistance and depth. The results of this paper are beneficial to future tire-terrain interaction research and implementation of tire model in full vehicle model. In addition, the developed equation will further allow to replace the rigid ring tire models by better non-linear relationships.

\section{Tire-terrain interaction}

The tire modeling and validation using Finite Element Analysis (FEA) technique is explained. Then, the smoothedparticle hydrodynamics (SPH) technique to model soft terrains is discussed. Finally, the tire-terrain interaction algorithm and the rolling resistance model set-up is explained.

\subsection{FEA tire model}

The FEA truck tire used in this research is modeled in Pam-Crash a multiphysics simulation software package and shown in Fig. 2. Furthermore, the FEA truck tire model developed in Pam-Crash is validated in static domain by performing the vertical stiffness and footprint tests and in dynamic domain by performing the drum-cleat test.

The tire's vertical stiffness, $k_{\text {tot }}$, is calculated during the vertical stiffness test. The truck tire is strained in all
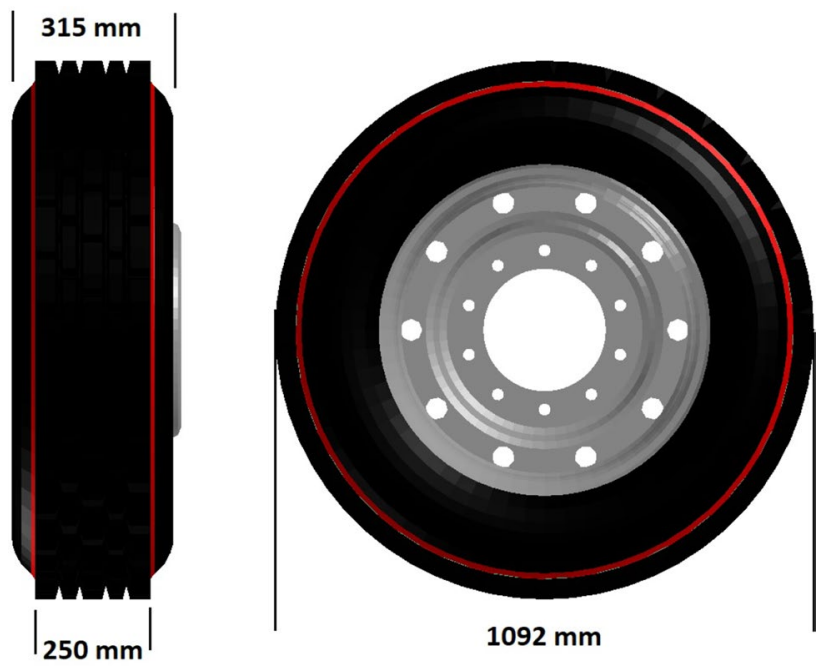

Fig. 2 315/80R22.5 FEA truck tire dimensions [10] 
translational and rotational directions apart from the vertical translational direction. The vertical rest starts by applying a low rate ramp vertical load to the center of the tire resulting in deformation. The relationship between the applied vertical load and the tire's center deflection is computed and the tire vertical stiffness, $k_{\text {tot }}$, is calculated using Eq. 1. The tire spring rate is the validated against provided manufacturer data.

$k_{\text {tot }}=\frac{\Delta \text { load }}{\Delta \text { deflection }}$

The contact patch of the tire with the ground is computed using the static footprint test as shown in Fig. 3. The same test procedure used in the vertical stiffness test is adapted in this test as well. Nonetheless, the contact area of the tire with the ground is recorded and compared to measured data at a rated inflation pressure of $758 \mathrm{kPa}(110 \mathrm{psi})$. The measured contact area provided from measured data is about $450 \mathrm{~cm}^{2}$ [11], while the calculated contact area is $498 \mathrm{~cm}^{2}$, with a difference less than $10 \%$.

The radial tire stiffness causes the tire radius to vary depending on the material characteristics and the tire inflation pressure. The drum-cleat test is used to compute the first mode frequency. The truck tire is excited over rigid circular drum with a cleat on top [12]. The vertical forces are computed and then the Fast Fourier Transformation (FFT) algorithm is deployed to convert the fores into frequency domain. The computed first model of vibration is then validated against measured data [12].

The predicted FEA truck tire vertical stiffness, $k_{\text {tot }}$ in $\mathrm{kN} / \mathrm{m}$ and the first mode of vibrations in $\mathrm{Hz}$ at an applied vertical load of $27 \mathrm{kN}$ (6000 lbs) and several inflation pressures are shown in Table 1.
Table 1 FEA truck tire predicted parameters at $27 \mathrm{kN}$ and different inflation pressures [11]

\begin{tabular}{lllll}
\hline Inflation pressure & 380 & 587 & 759 & $\mathrm{kPa}$ \\
Vertical stiffness, $k_{\text {tot }}$ & 575 & 818 & 9994 & $\mathrm{kN} / \mathrm{m}$ \\
Frequency first mode & 46 & 53 & 57 & $\mathrm{~Hz}$ \\
\hline
\end{tabular}

\subsection{SPH terrain model}

The particle is described as a sphere centred on the centre of mass, each particle has a radius, a mass, and a velocity. The discretized conservation equations governs the change of particle characteristics. Each particle also has three Degrees of Freedom (DOF), namely the centre of mass of the particle, the volume of the particle, and the domain of influence [13].

The smoothing length, $h$, is used to define the distance between two consecutive particles, while the minimum smoothing length is defined as $H_{\min }$ and the maximum smoothing length is defined as $H_{\max }$. The Anti-Crossing force (ETA) relationship is shown in Eq. 2, where $\epsilon$ is the relative strength which is generally less than $0.5, \rho$ is the density of the particles and $W_{i j}$ is the weighing function [11].

$E T A=u_{i}+\epsilon \sum_{j} m_{j} \frac{u_{j}-u_{i}}{\frac{1}{2}\left(\rho_{i}+\rho_{j}\right)} W_{i j}$

In this research, several terrains are modeled and calibrated to predict the tire-terrain interaction. The terrain modeled include soils such as dry and moist sand, clayey soil, dense sand and snow which are modeled using the isotropic-elastic-plastic-hydrodynamic relationship [10, $11,14]$, while other terrains includes the water to model flooded surface [15-17]. Furthermore, the moisture effect
Fig. 3 Normal nodal velocity acting on the tire-road contact area at $27 \mathrm{kN}$ vertical load and $758 \mathrm{kPa}$ inflation pressure

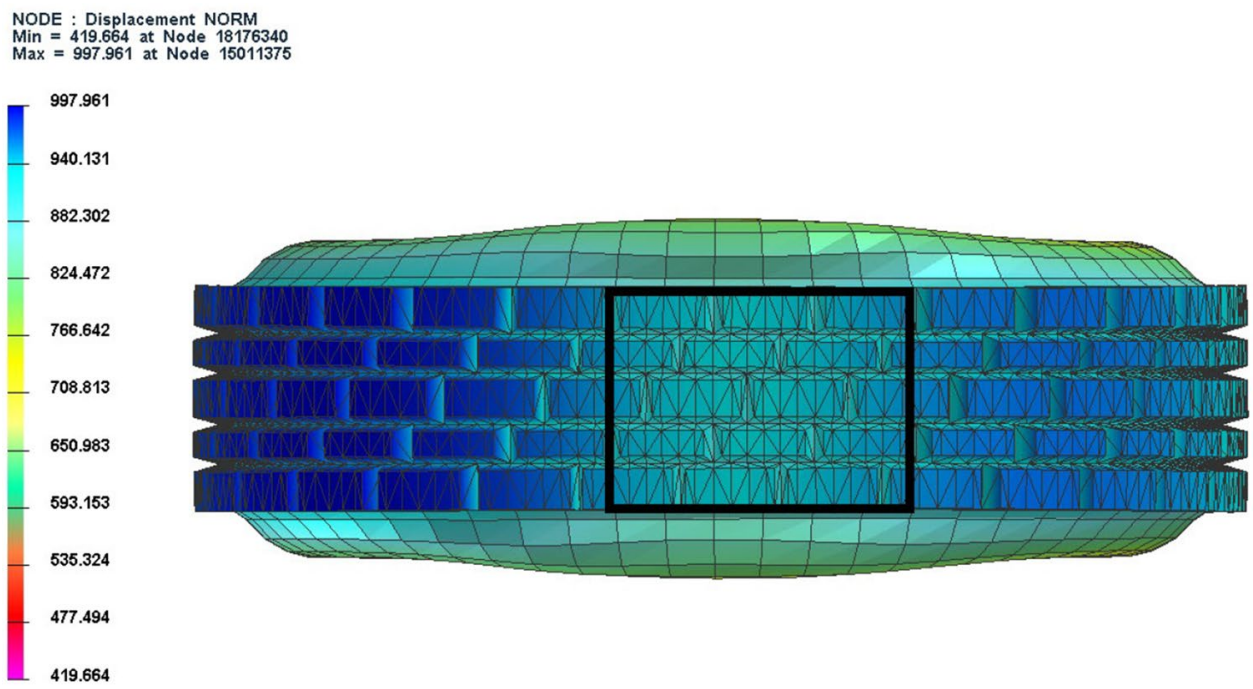


of the soils is also taken into account by modeling moist sand and sandy loam with different moisture content [18].

Furthermore, Murnaghan equation of state for solid elements is used to model water and flooded surfaces. The equation of state requires the input of the density $\rho$, the bulk coefficient $B$, and the exponent $\gamma$, in addition to viscosity coefficients. The Murnaghan equation of state relationship is described in Eq. 3, the exponent $\gamma$ is the density ratio exponent which is generally equal to 7 , and the ratio $\rho / \rho_{0}$ is the current to the initial mass densities. Equation 4 is used to determine the coefficient $B$ which is related to the speed of sound.

$p=p_{0}+B\left(\left(\frac{\rho}{\rho_{0}}\right)^{\gamma}-1\right)$

$B \geq 100 \rho_{0} \frac{V_{\max }^{2}}{\gamma}$

The isotropic-elastic-plastic-hydrodynamic material was used to model the soil behavior. Equation 5 shows the Equation of State (EOS) for isotropic-elastic-plastic-hydrodynamic material which governs the pressure-volume relationship at low pressure. Where, $c_{0}$ to $c_{6}$ are EOS coefficients, $\mu$ is the ratio of current over initial mass density and is written as $\mu=\rho / \rho_{0}-1$, and $E_{i}$ is the internal energy [13]. The material properties used in this research are obtained from published terramechanics research, these properties are implemented in the soil definition to calibrate the soil characteristics.

$p=c_{0}+c_{1} \mu+c_{2} \mu^{2}+c_{3} \mu^{3}+\left(c_{4}+c_{5} \mu+c_{6} \mu^{2}\right) E_{i}$

The pressure-sinkage and the shear-strength tests are performed to calibrate the modeled soil. The results of the modeled soil are calibrated against published terramechanics data.

\subsubsection{Pressure-sinkage test}

A constant pressure is applied to a circular plate of $150 \mathrm{~mm}$ radius, the plate is located on top of a $800 \times 800 \times 800 \mathrm{~mm}$ box filled with soil domain shown in Fig. 4. The soil domain is exposed to a range of pressures between 0 to $200 \mathrm{kPa}$ and the sinkage of the plate is recorded for each pressure at steady state. The results obtained from the pressure-sinkage test are calibrated against Bekker's relationship presented in Eq. 6 . Where $p$ is the applied pressure in $\mathrm{kPa}, b$ is radius of the circular plate in $\mathrm{mm}, z$ is the plate sinkage in $\mathrm{mm}$, and $n, k_{c}$, and $k_{\theta}$ are soil parameters obtained from published terramechanics research.

$p=\left(\frac{k_{c}}{b}+k_{\theta}\right) z^{n}$.

\subsubsection{Shear-strength test}

A soil domain is filled inside a rectangular box, as displayed in Fig. 5. The box consists of three components the top pressure plate where pressure is applied, the upper box which is the sliding component and the fixed bottom box which is fixed in all directions. A constant pressure is applied to the centre of the top pressure plate, then a $10 \mathrm{~m} / \mathrm{s}$ displacement is applied to the upper box and the top plate. The shear force is recorded and the shear-stress relationship is obtained. The shear characteristics of the soil obtained form the shear-stress relationship is calibrated against Janosi and Hanamoto exponential function shown in Eqs. 7 and 8.

Fig. 4 Pressure-sinkage test [10]

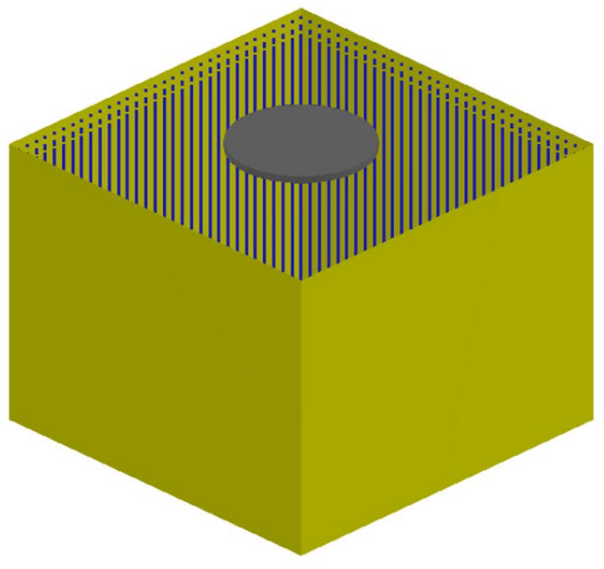

(a) intial stage

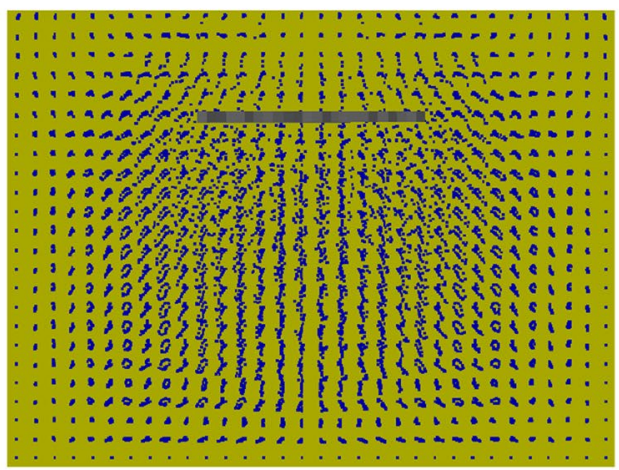

(b) final stage

SN Applied Sciences 

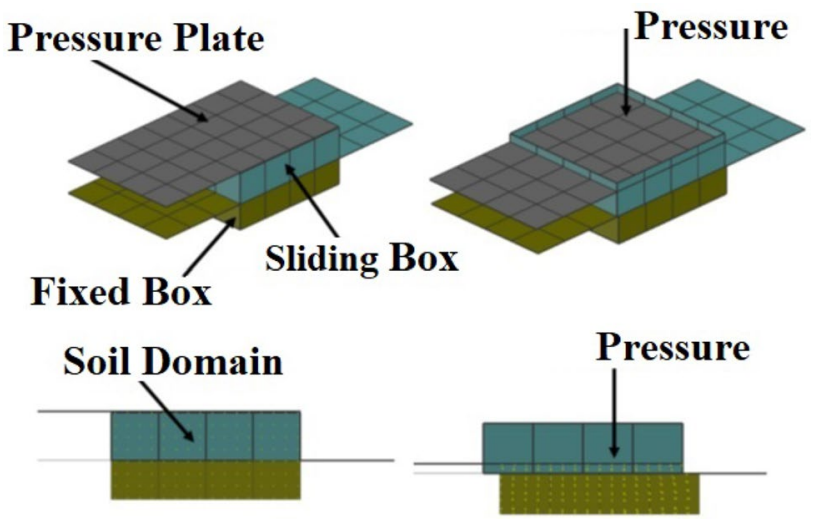

Fig. 5 Shear-strength test [10]

$\tau=\tau_{\max }\left(1-e^{(-j / k)}\right)=(c+p \tan \phi)\left(1-e^{(-j / k)}\right)$

Mohr-Coulomb failure criterion shown in Eq. 8 is used to determine the maximum shear $\tau_{\max }$, where $\mathrm{c}$ is the soil cohesion in $\mathrm{kPa}$ and $\phi$ is the angle of shear resistance in degrees obtained from published terramechanics data.

$\tau_{\max }=c+p \tan \phi$

The calibration procedure requires the repetition of both tests using exact material properties to obtain realistic soil characteristics. In the course of the calibration and validation, the material properties are repeatedly adjusted, and the pressure-sinkage and shear-strength calibration tests are repeated until the simulation results and the terramechanics data are in best agreement.

\subsection{Rolling resistance analysis}

The rolling resistance analysis is performed under different operating conditions and several terrains to determine the effect on the tire-terrain interaction as shown in Fig. 6. The results of the simulations are then used in a ANN-GA algorithm to develop a numerical relationship between the rolling resistance coefficient and the terrain parameters and operating conditions.

The rolling resistance simulation is common for all operating conditions. A pressure is applied to the tire components to obtain a specified inflation pressure, then a rapid constant vertical load is applied to the centre of the tire. The tire is then left on the terrain to stabilize. Later, a constant linear longitudinal velocity is applied to the centre of the tire. The tire then rolls over the terrain for about $1.5 \mathrm{~s}$ to obtain steady forces at the contact patch. The rolling resistance coefficient, $f_{r}$, is

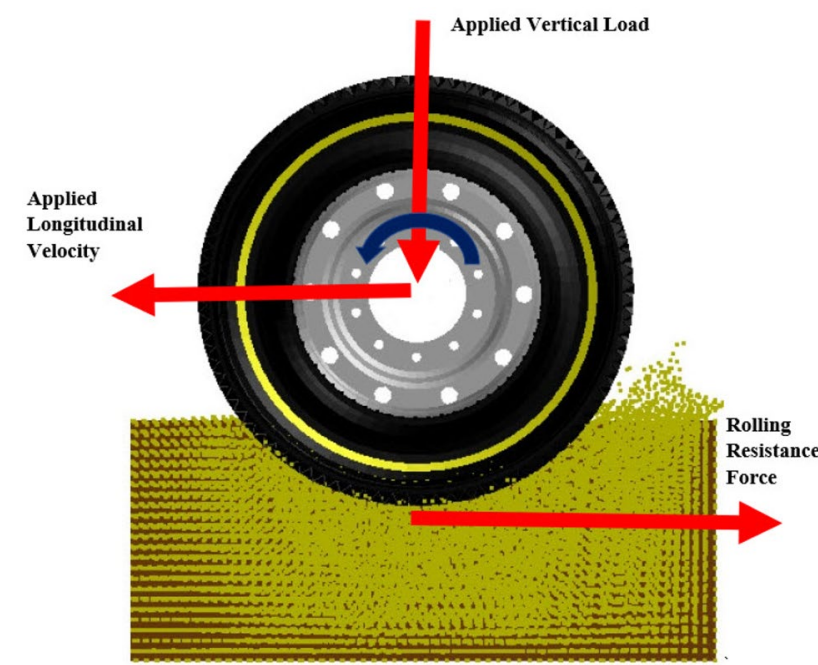

Fig. 6 Schematic of a tire during rolling resistance test over dry sand

then calculated as shown in Eq. 9, where $F_{x}$ and $F_{z}$ are the average forces at steady state, in the longitudinal and vertical directions, respectively.

$f_{r}=\frac{F_{x}}{F_{z}}$

The performed simulation to predict rolling resistance is repeated at several terrains including flooded surface, snow, dry and moist sand, clayey soil, dense sand, and snow. The obtained results are validated and published $[10,11,17]$. The simulations are repeated at different operating conditions such as; inflation pressures (379 $\mathrm{kPa}, 586 \mathrm{kPa}$ and $758 \mathrm{kPa}$ ); applied vertical loads $(13 \mathrm{kN}, 27 \mathrm{kN}$, and $40 \mathrm{kN})$; and tire speed ranging between 10 and $100 \mathrm{~km} / \mathrm{h}$.

Figure 7 shows the rut depth in the several terrains for the tire running at $10 \mathrm{~km} / \mathrm{h}$ speed, $27 \mathrm{kN}$ vertical load and $586 \mathrm{kPa}$ inflation pressure. It is observed that rut depth left in soil depends on the terrain type and moisture content, for instance the rut left in dry sand around $225 \mathrm{~mm}$ which is less than that left in clayey soil of around $157.5 \mathrm{~mm}$. Also, in comparison between dry sand which has a rut depth of $225 \mathrm{~mm}$ and $10 \%$ moist sand which has a rut depth of $344 \mathrm{~mm}$, the moist sand has a greater rut depth.

In the case of snow, the rut depth recorded was around $300 \mathrm{~mm}$ this is due to the fact that the tire in snow causes a splash phenomenon which causes some particles of the snow to splash away of the box. It is also noticed that in cases such as clayey soil and snow due to the high sinkage of the tire in the terrain the front zone of the terrain also display before the tire is in contact, this is also referred to as bulldozing effect. 


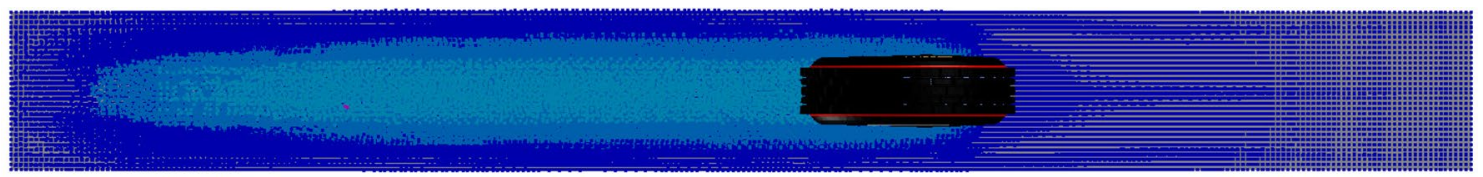

(a) dry sand, rut depth of $225 \mathrm{~mm}$

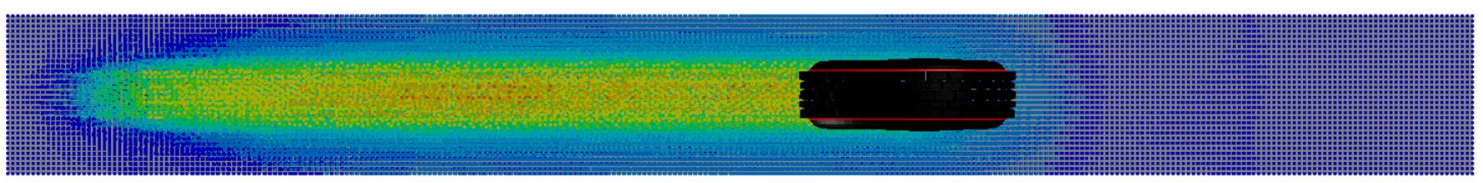

(b) dense sand, rut depth of $61 \mathrm{~mm}$

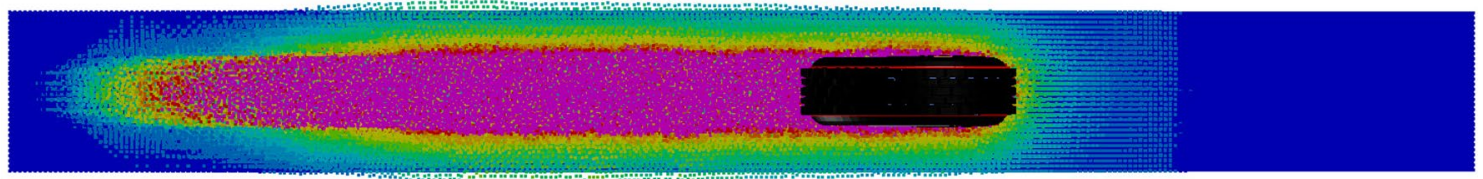

(c) clayey soil, rut depth of $157.5 \mathrm{~mm}$

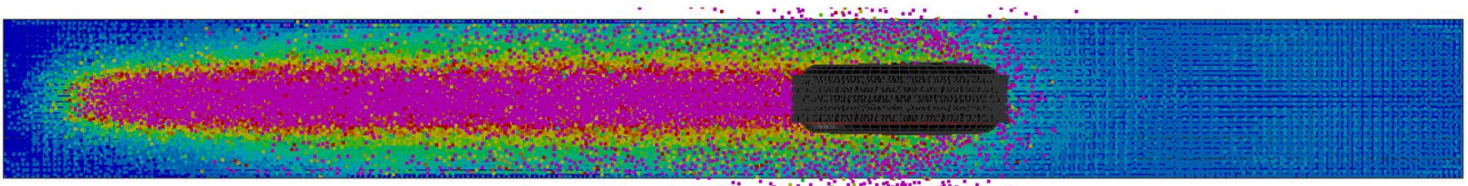

(d) $10 \%$ moist sand, rut depth of $344 \mathrm{~mm}$

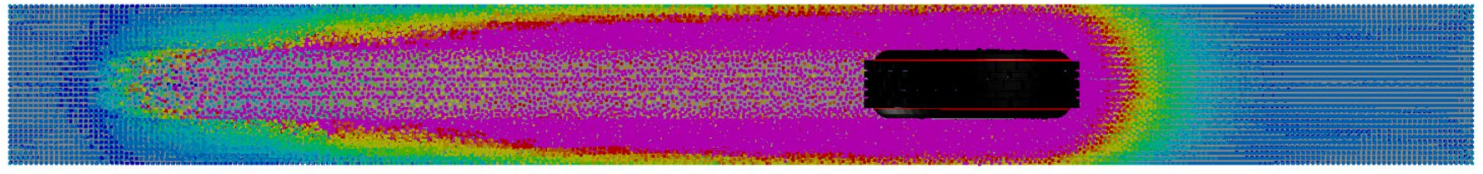

(e) snow, rut depth of $300 \mathrm{~mm}$

Fig. 7 Rut depth left in different terrains for tire running at $10 \mathrm{~km} / \mathrm{h}$ and $0.5 \mathrm{~s}$

\section{Analytical results}

The ANN and the GA methods are used to numerically relate the rolling resistance coefficient of the truck tire running over the previously mentioned operating conditions. The purpose of this research is to evaluate the results obtained from both methods, and to develop a relationship between the rolling resistance coefficient, $f_{r}$, the terrain parameters and the operating conditions in order to determine the rolling resistance coefficient without the need to perform simulations.

The terrains used include dry sand, dense sand, clayey soil, water, snow, sandy loam with moisture content and moist sand. The terrains are presented using the cohesion, $C$, in $\mathrm{kPa}$ angle of shear resistance, $\phi$, in degrees and depth, $D$, in $\mathrm{m}$ while the tire operating conditions are presented using the inflation pressure, $P$, in $\mathrm{kPa}$ vertical load, $L$, in $\mathrm{kN}$ and speed, $V$ in $\mathrm{m} / \mathrm{s}$. Thus, each output rolling resistance coefficient, $f_{r}$, value is associated with six inputs, the input vector $X$ is defined as show in Eq. 10 .

\section{SN Applied Sciences}

A SPRINGER NATURE journal

$$
X^{T}=\left[\begin{array}{llllll}
L & P & \vee & C & \phi & D
\end{array}\right] .
$$

\subsection{Artificial neural network}

Two-layer of fully-interconnected feed-forward ANN are used as shown in Fig. 8. A fully-interconnected ANN indicates that each input is connected to each neuron and the data is processed from left to right which is the forward direction. The parameter $b_{i}$ is the bais which is shown in Fig. 1.

In the case of a single neuron, the relationship between the input and the output is shown in Eq. 11, where $f$ is the activation function performing the summation of weighted inputs. Equation 11 can be expressed in matrix form as shown in Eq. 12. Where $W$ and $P$ are expressed as shown in Eqs. 13 and 14, respectively.

$y=f\left(w_{1} p_{1}+w_{2} p_{2}+w_{3} p_{3}+\cdots+w_{n} p_{n}+b\right)$ 
Fig. 8 Diagram of a two-layer fully-interconnected feedforward ANN

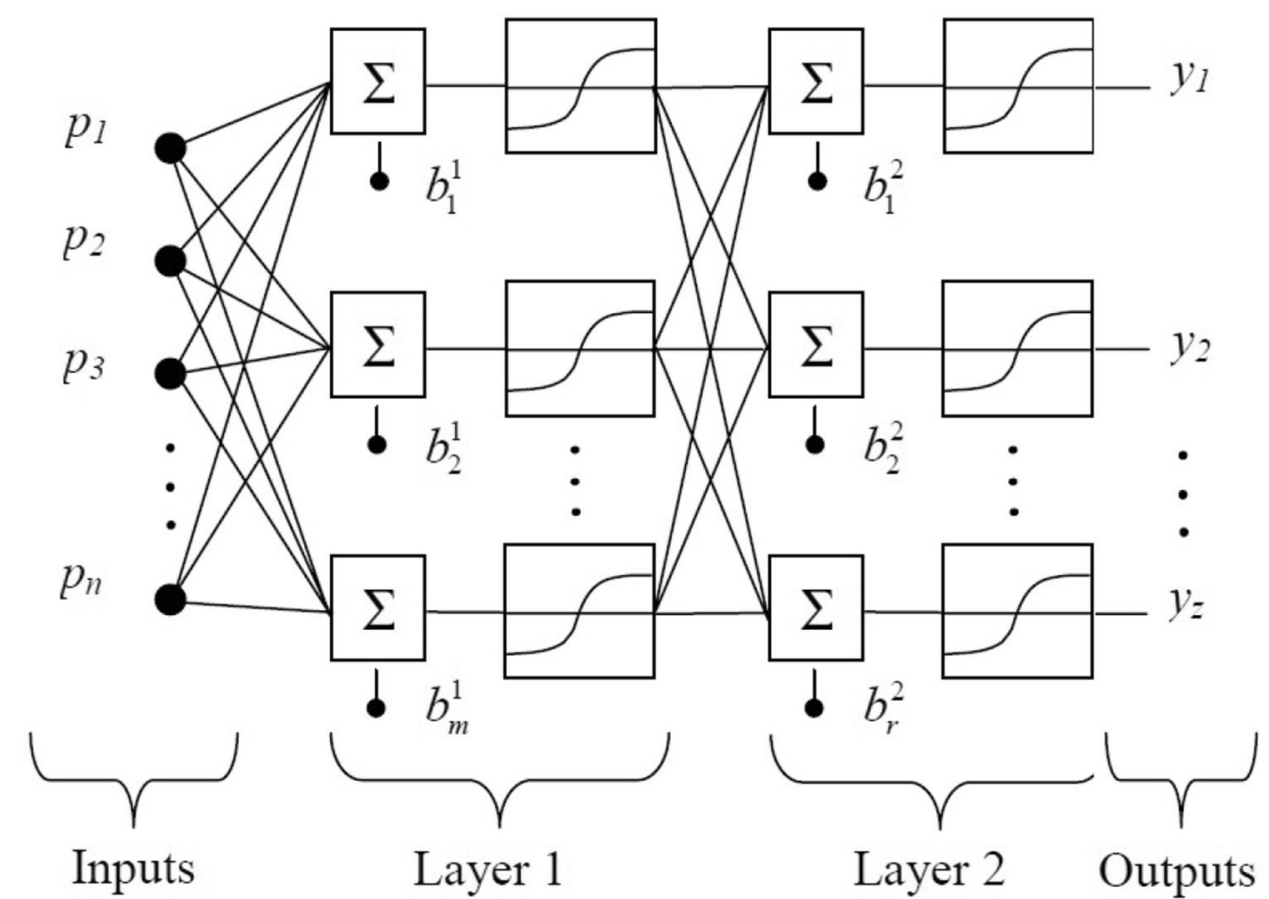

$y=f(W P+b)$

$W=\left[\begin{array}{lllll}w_{1} & w_{2} & w_{3} & \ldots & w_{n}\end{array}\right]$

$P=\left[\begin{array}{lllll}p_{1} & p_{2} & p_{3} & \ldots & p_{n}\end{array}\right]^{T}$

In a general form for an ANN with $k$ number of layers the desired output is written as shown in Eq. 15.

$\begin{aligned} Y^{k}= & f^{k}\left(w^{k} f^{k-1}\left(W^{k-1} f^{k-2}\left(W^{k-2} f^{k-3}\left(W^{k-3}(\ldots)+B^{k-3}\right)\right.\right.\right. \\ & \left.\left.\left.+B^{k-2}\right)+k^{k-1}\right)+B^{k}\right)\end{aligned}$

where the matrix $Y^{k}, B^{k}$, and $W^{k}$ are defined in Eqs. 16, 17, and 18 , respectively.

$Y^{k}=\left[\begin{array}{lllll}y_{1}^{k} & y_{2}^{k} & y_{3}^{k} & \cdots & y_{z}^{k}\end{array}\right]^{T}$

$B^{k}=\left[\begin{array}{llll}b_{1}^{k} & b_{2}^{k} & b_{3}^{k} \ldots & b_{z}^{k}\end{array}\right]^{T}$

$w^{k}=\left[\begin{array}{ccccc}w_{1,1}^{k} & w_{1,2}^{k} & w_{1,3}^{k} & \ldots & w_{1, n}^{k} \\ w_{2,1}^{k} & w_{2,2}^{k} & w_{2,3}^{k} & \ldots & w_{2, n}^{k} \\ w_{3,1}^{k} & w_{3,2}^{k} & w_{3,3}^{k} & \ldots & w_{3, n}^{k} \\ w_{m, 1}^{k} & w_{m, 2}^{k} & w_{m, 3}^{k} & \ldots & w_{m, n}^{k}\end{array}\right]$

The ANN is implemented in MATLAB using the "Neural Net Fitting" tool, the number of layers was chosen to be two, with tan-sigmoid activation function in the hidden layer and linear neurons at the output layer which is a commonly used function approximation. The number of neuron in each layer was set to 10. Generally, the equation that describes the input-output mapping for an ANN with tan-sigmoid neurons derived from Eq. 12 in the hidden layer and linear neurons in the output is shown in Eq. 19. Where $Y$ is the output vector which is in this case the RRC $[1 \times 1], X$ is the input vector which is in this case $[6 \times 1], L W$ is the first layer weight matrix with size $[10 \times 6], I W$ is the second layer weight matrix with size $[1 \times 10], b_{1}$ and $b_{2}$ are the first and second layer bias vector. It should be noted that since 10 neurons were deployed during the definition process the vector $b_{1}$ has the size $[10 \times 1]$ and the vector $b_{2}$ is $[1 \times 1]$.

$Y=L W\left(\operatorname{tansig}\left(I W X+b_{1}\right)\right)+b_{2}$

The tansig function is defined below:

$\operatorname{tansig}\left(I W X+b_{1}\right)=\frac{2}{1+e^{-2\left(I W X+b_{1}\right)}}-1$

After collecting all the rolling resistance coefficient data from simulations an input of [ $6 \times 147]$ and an output of $[1 \times 147]$ were imported into MATLAB "Neural Net Fitting" toolbox. Consequently, an ANN training was performed and the bias vector and weight matrix were determined. Details about the ANN fitting and equation are given in 
"Appendix". Figure 9 shows the variation of the observed rolling resistance coefficient as a function of the predicted.

The mean square error (MSE) was computed to be $5 e^{-5}$ and the R-square goodness of fit is 0.9974 . It is clearly observed that the observed and predicted rolling resistance coefficients falls within the same values and are very close to each others over the whole range. The observed and predicted rolling resistance coefficients are in the range of the line with equation $y=x$ which shows the perfect fitting between both values. It is concluded that the ANN equation successfully predicts the rolling resistance coefficient for the range of the 6 inputs (inflation pressure, vertical load, terrain cohesion, terrain shear resistance, terrain depth, and tire speed).

\subsection{Generic algorithm}

The GA is utilized in this research to find the relationship between various parameters. A basic GA has 10 simple steps, first a chromosome of a fixed length is used to represent the problem variable domain, then the size of the chromosome population, $N$ is chosen. Second, the fitness function is defined to measure the fitness of each individual chromosome. Third, an initial population of chromosomes of size $\mathrm{N}$ is randomly generated. Fourth, the fitness of each individual chromosome is computed. Fifth, a pair of chromosomes is then selected for mating from the current population, the selection process is based on a fitness probability. Sixth, a pair of offspring chromosomes is created by implementing the crossover and mutation. Seventh, created offspring chromosomes are placed in the new population and fifth step is repeated until the new chromosome population size is equal to the initial population size. Then, the initial chromosome population is replaced with the new offspring one. Finally, all steps from step four are repeated until the termination criterion is fulfilled.

The fitness functions are well known in the optimization process, the optimization problem is generally defined by Eq. 21 , where $\phi_{i}^{t}$ is the penalty function at the ith string in the tth generation, $X_{i}^{t}$ is the vector of the design variable and $N$ as mentioned before is the population size.

$\phi_{i}^{t}=f\left(X_{i}^{t}\right)+\sum_{j=1}^{m} \beta_{j} \max \left(0, g_{i}\left(X_{i}^{t}\right)\right) \quad(i=1 \ldots N)$

Implementing the same technique used for ANN and using the same inputs and outputs obtained from simulations an equation relating the input parameters to the rolling resistance coefficient, $f_{r}$, is generated using Eureqa a modeling engine. Equation 22 describes the rolling resistance coefficient, $f_{r}$ as a function of the input parameters, where $k_{1}, k_{2}, k_{3}$ and $k_{4}$ are constant equal to $0.0876,0.167,1.08 \mathrm{e}-5$ and 0.001 , respectively.

$f_{r}=k_{1} V * D+k_{2} D^{2}+k_{3} L * P * D^{2}+k_{4} \frac{L}{\phi-12.4}+\frac{\phi D}{L * C^{2}}$

Figure 10 presents the observed as a function of the predicted rolling resistance coefficient. The R-square goodness measures how close the data are to the fitted regression line as shown in Eq. 23.
Fig. 9 Observed as a function of the predicted rolling resistance coefficient for an R-square fitness

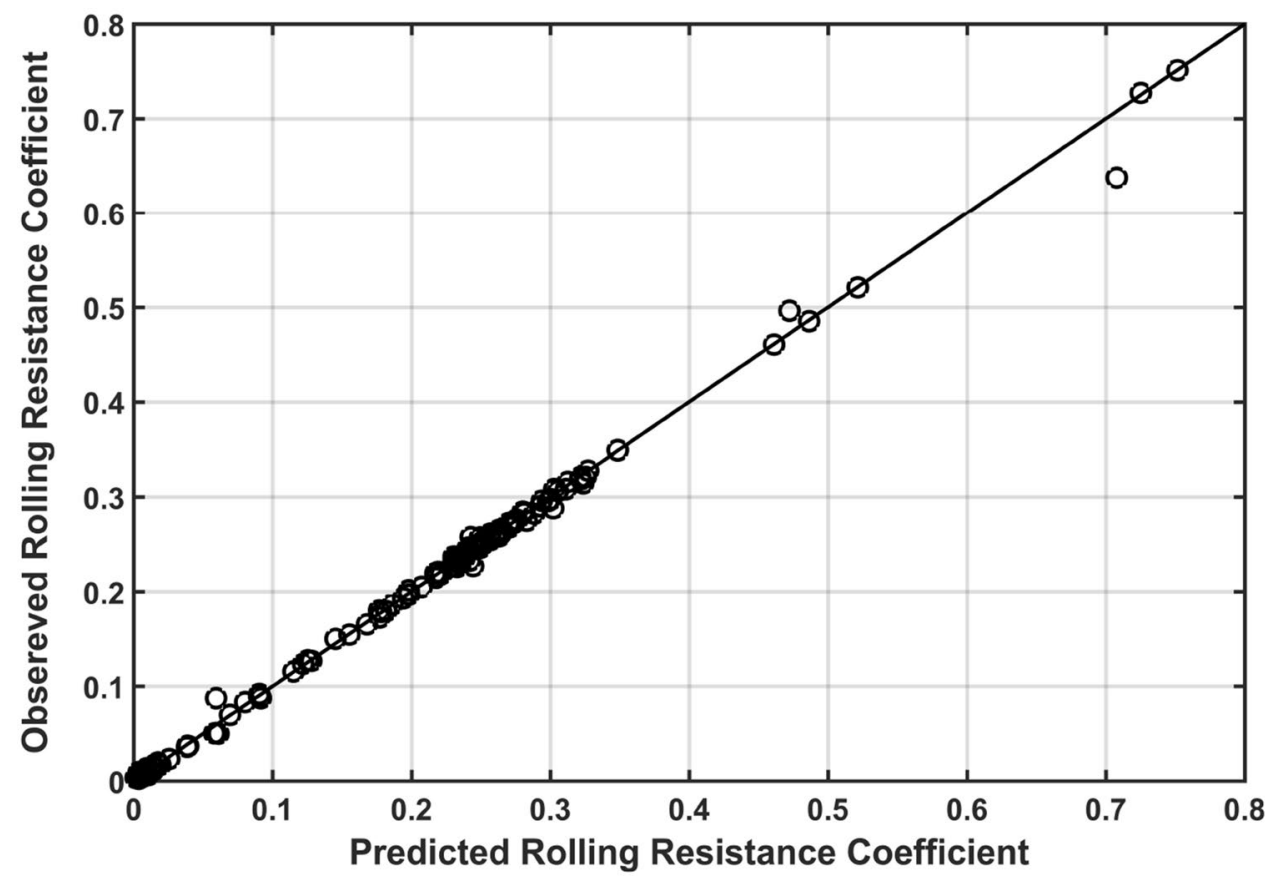


Fig. 10 Observed as a function of the predicted rolling resistance coefficient for an R-square fitness

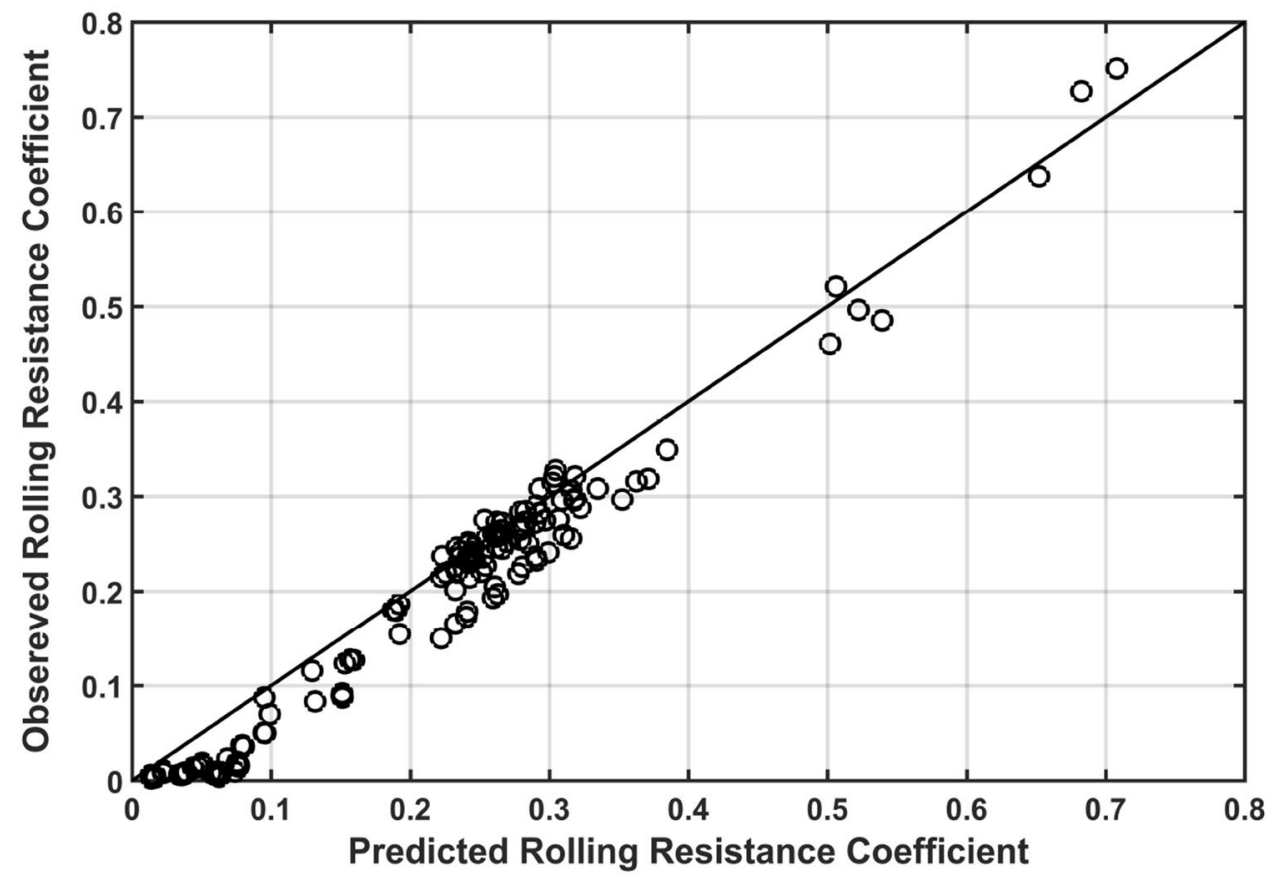

For example a value of 0 imply that the model demonstrate none of the variability of the response data around its means, while a value of 1 imply that the model demonstrate total variability of the response data around the mean. Thus generally the higher the $R^{2}$ goodness the better. The calculated R-square of Eq. 22 is 0.94 .

$R^{2}=\frac{\text { Explained variation }}{\text { Total variation }}$

The Mean Absolute Error (MAE) is used to minimize the mean of the absolute value of residual errors and is defined in Eq. 24, $n$ is the number of data points, $y$ is the hydroplaning speed input and $f(x)$ is the hydroplaning speed predicted by the equation.

$M A E=\frac{1}{n} \sum_{i=1}^{n}|y-f(x)|$

The MAE calculated for Eq. 22 using Eq. 24 is 0.027. Furthermore, the Mean Squared Error (MSE) is used to minimize the mean of the squared residual errors and is defined in Eq. 25.

$M S E=\frac{1}{n} \sum_{i=1}^{n}(y-f(x))^{2}$

The calculated MSE is 0.0012 based on Eq. 25. It is noticed that the observed and predicted rolling resistance coefficients falls within a similar range and clearly lies in the range of the straight line with equation $y=x$. However, for a low rolling resistance coefficient value less that 0.1 the results are slightly scattered.

\subsection{Testing of algorithms performance}

In comparison between Figs. 9 and 10 it is observed that the fitting of the observed versus the predicted rolling resistance coefficient of the the neural network is better than that of the genetic algorithm. However, on the other side the complexity of neural network equation mentioned in Eq. 34 is way higher than that of the genetic algorithm equation mentioned in Eq. 22. Thus a compromise between the complexity of the equation and the fitness should be made depending on the application purpose.

To further estimate the performance of the ANN and GA algorithms, Fig. 11 is presented. Figure 11 shows the variation of the rolling resistance coefficient as a function of the tire speed for a tire running over $200 \mathrm{~mm}$ snow depth, $27 \mathrm{kN}$ vertical load and $586 \mathrm{kPa}$ inflation pressure for simulations, ANN and GA algorithms. All three lines shows the same pattern as the tire speed increases the rolling resistance coefficient increases for a constant depth, load and inflation pressure. However, the ANN algorithm better predicts the simulation results in comparison to the GA predictions. Overall, the GA predictions are at a maximum error of 0.06 .

Furthermore, the variation of the rolling resistance coefficient at $27 \mathrm{kN}$ vertical load, $586 \mathrm{kPa}$ inflation pressure, $10 \mathrm{~km} / \mathrm{h}$ tire speed and a soil depth of $585 \mathrm{~mm}$ as a function of the soil cohesion is represented in Fig. 12. Generally, the rolling resistance coefficient for all three curves 
Fig. 11 rolling resistance coefficient as a function of tire speed for simulations, ANN and $\mathrm{GA}$ algorithms

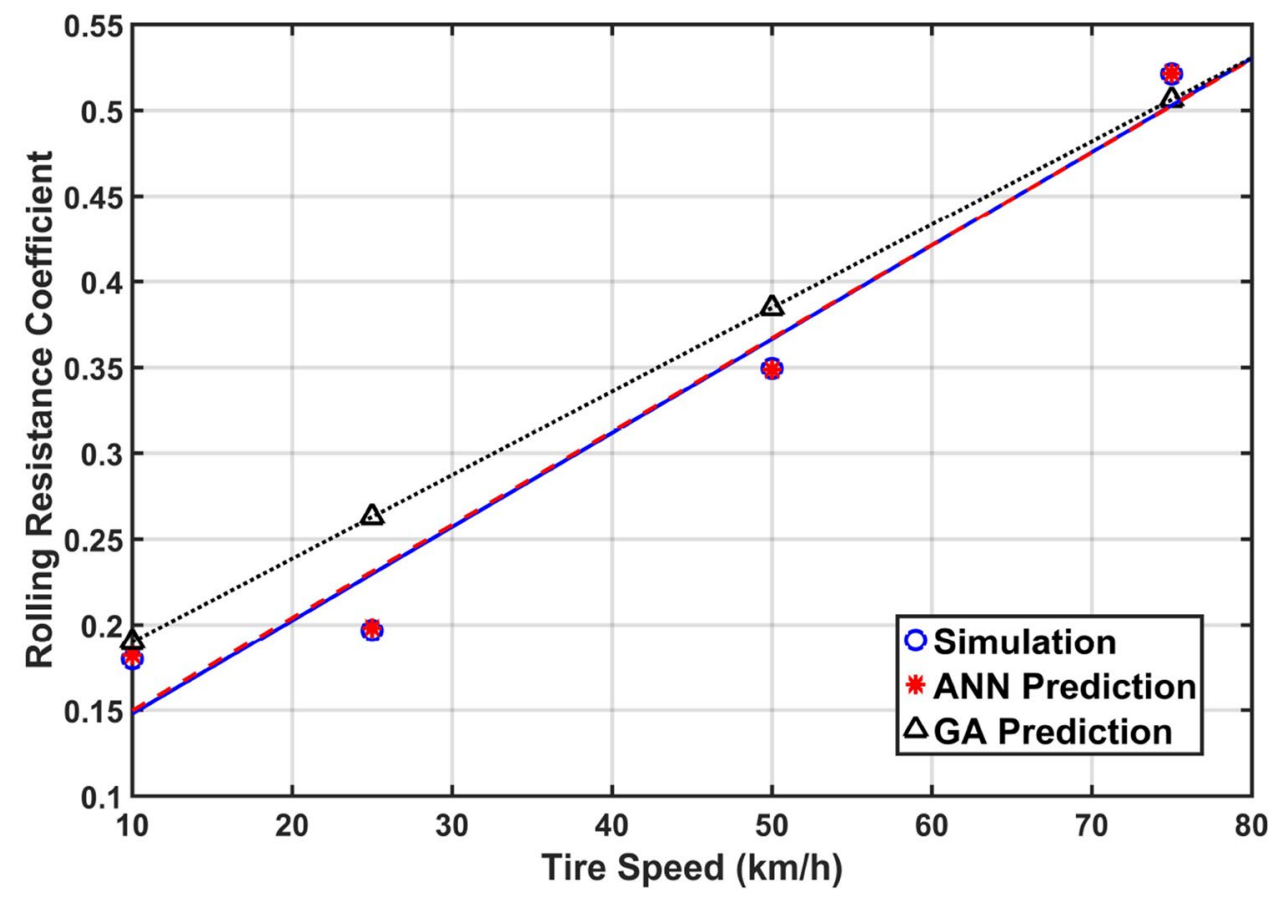

Fig. 12 Rolling resistance coefficient as a function of soil cohesion for simulations, ANN and GA algorithms

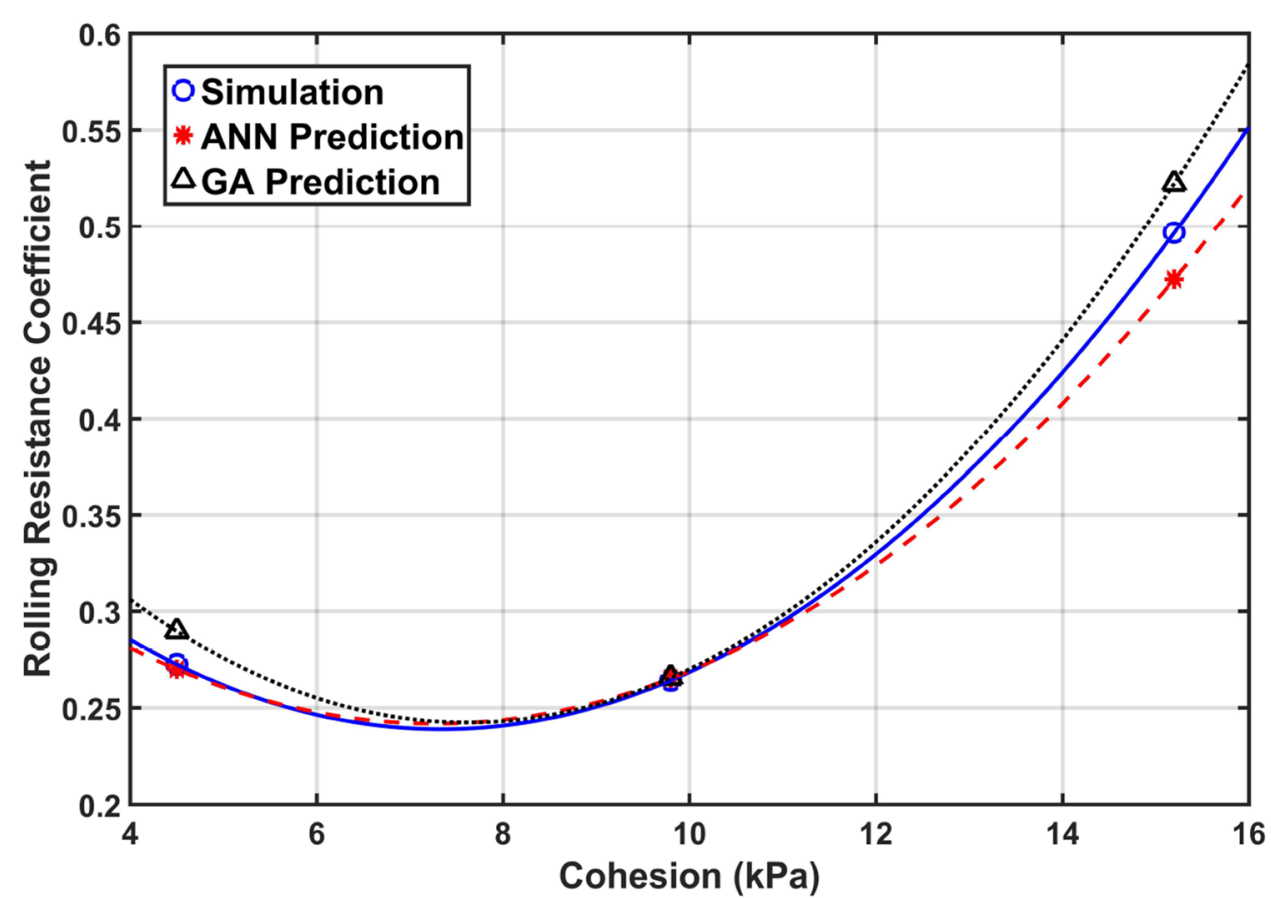

exhibits the same trend. However, for a low soil cohesion the ANN predictions are closer to those of the simulations in comparison to that of the GA predictions. At higher cohesion such as $15 \mathrm{kPa}$ both ANN and GA are within a maximum error of 0.025 .

The variation of the rolling resistance coefficient at $27 \mathrm{kN}$ vertical load, $586 \mathrm{kPa}$ inflation pressure, $10 \mathrm{~km} / \mathrm{h}$ tire speed and a soil depth of $585 \mathrm{~mm}$ as a function of the soil shear resistance angle is represented in Fig. 13. Generally, all three lines has the same trend, as the angle of shear resistance increases the rolling resistance coefficient reduces. Moreover, at low angle of shear resistance both ANN and GA have a maximum error of 0.03 , at high angle of shear resistance the three linear are coinciding. 
Fig. 13 Rolling resistance coefficient as a function of soil angle of shear resistance for simulations, ANN and GA algorithms

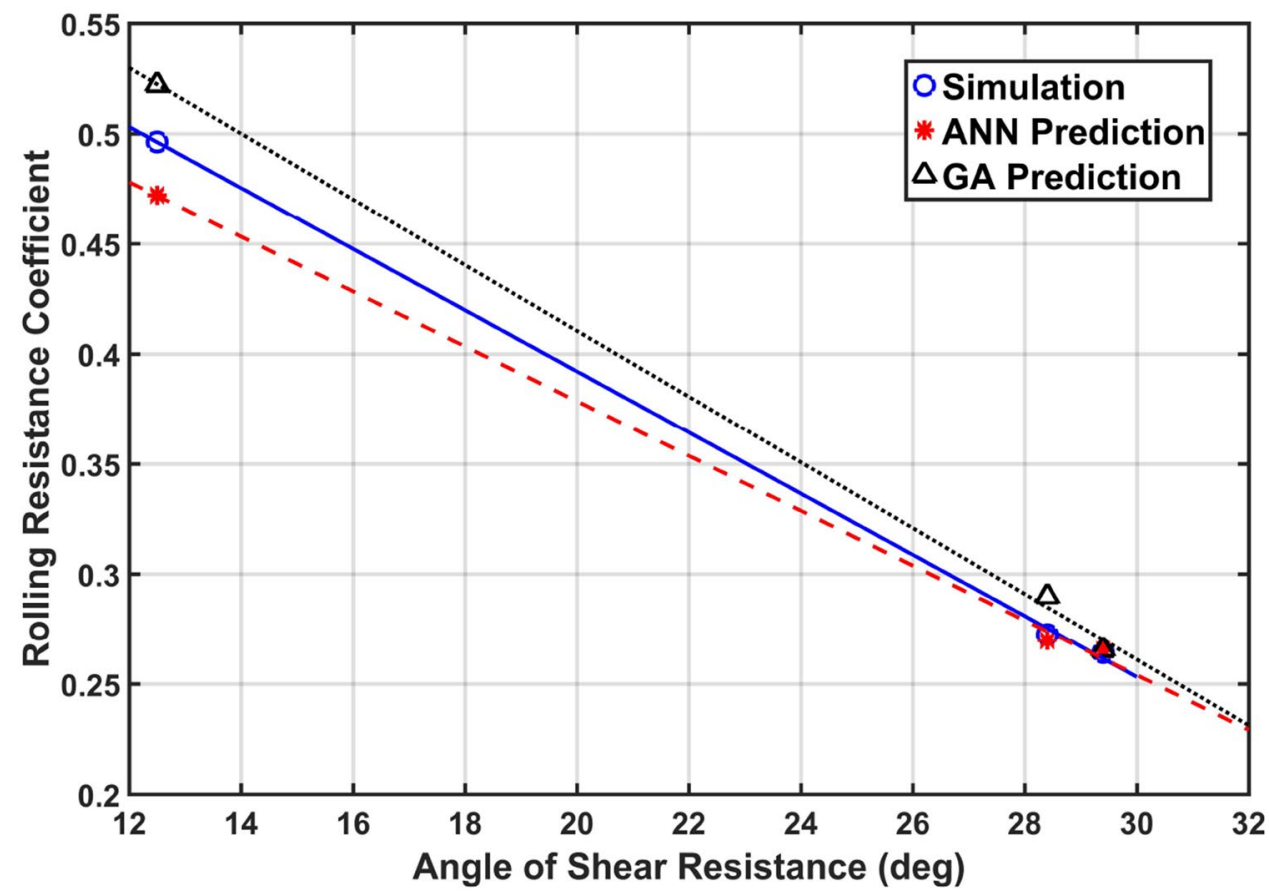

\section{Conclusions}

A Finite Element Analysis off-road tuck tire size 315/80R22.5 was modeled and validated. The rolling resistance coefficient of the truck tire running over various terrains at different operating conditions was computed. The terrains were modeled using smoothed-particle hydrodynamics technique and calibrated and validated against published terramechanics data. The various terrains included dry and moist sand, clayey soil, flooded surface, snow, dense sand and sandy loam with different moisture content. The different operating conditions included inflation pressures, vertical loads, and tire speeds.

The rolling resistance coefficient results obtained from several FEA-SPH simulations were used to built a numerical equation relating between the rolling resistance coefficient and the terrain parameters and operating conditions. The data collected was used as an input/output to the algorithms with the terrain parameters (cohesion, angle of shear resistance, depth) and the operating conditions (tire inflation pressure, applied vertical load and tire speed) as an input and the rolling resistance coefficient as an output.

Two relationships were built using the artificial neural network (ANN) and the genetic algorithm (GA) methods.
The mean square error of the ANN and GA algorithms was computed to be $5 e^{-5}$ and 0.0012 , respectively. While the R-square goodness of fit was 0.9974 for the ANN and 0.94 for the GA. It was concluded that the ANN has a better fitting in regards to the observed versus predicted rolling resistance coefficient, while the GA has a better numerical equation in terms of complexity.

The main concluding remarks are presented as follows:

- A hybrid FEA-SPH model to simulate the tire-terrain interaction was developed and validated.

- The rolling resistance coefficient of the truck tire running over different terrains at various operation condition were collected.

- Two relationships using the ANN and GA were developed to predict the rolling resistance coefficient as a function of operation conditions.

- The ANN developed relationship has a better fitting in regards to the observed versus predicted rolling resistance coefficient.

- The GA developed relationship has a better numerical equation in terms of complexity. 
The develop equation is based on the set of simulations performed and is limited to the truck tire used. It would be beneficial for further studies to test and validate the equation on different tires and various operating conditions.

Acknowledgements The authors express their gratitude to NSERC Discovery Grant for funding this study and to Volvo Group Trucks Technology for their continuous support during the course of this study.

\section{Compliance with ethical standards}

Conflict of interest The authors declare that they have no conflict of interest.

\section{Appendix}

The bias and weight of the ANN were determined as follows:

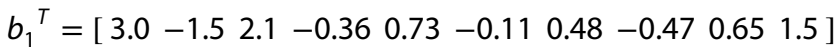

$b_{2}=-2.3$

$I W=\left[\begin{array}{cccccc}0.058 & -0.023 & -0.011 & -1.5 & 3.9 & -1.2 \\ 0.81 & 0.069 & 2.1 & -0.68 & -1.0, & 1.9 \\ -0.68 & -0.043 & -0.72 & 1.7 & -0.32 & -0.2 \\ -0.033 & -0.085 & -1.1 & 1.6 & -0.048 & -2.0 \\ 0.19 & -0.58 & -1.1 & -0.74 & -1.1 & -1.9 \\ 0.11 & -0.029 & 0.69 & 1.5 & 2.2, & 0.65 \\ -0.079 & 2.8 e-3 & -1.8 & -1.4 & -3.5 & -1.5 \\ -0.61 & 0.018 & 1.0 & -0.032 & -0.011 & -0.54 \\ 0.84 & -0.023 & -1.8 & 0.67 & 0.43 & 0.48 \\ -1.0 & -0.02 & -1.2 & 3.2 & 2.2 & 1.4\end{array}\right]$

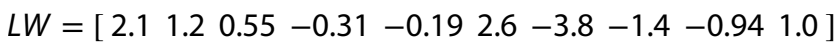

Let $M$ be defined as show in Eq. 30, in addition the tansig is a ANN transfer function that calculates the layer's output from its net input as defined as shown in Eq. 31.

$M=I W X+b_{1}$

$\operatorname{tansig}\left(I W X+b_{1}\right)=\frac{2}{1+e^{-2\left(I W X+b_{1}\right)}}-1$

Substituting $I W, b_{1}$ and the input $X$ Eq. 30 is rearranged and presented in 32 .

$M=\left[\begin{array}{c}0.058 L-1.2 D-1.5 C-0.023 P-0.011 V+3.9 \phi+3 \\ 1.9 D-0.68 C+0.81 L+0.07 P+2.1 V-\phi-1.5 \\ 1.7 C-0.2 D-0.68 L-0.043 P-0.72 V-0.32 \phi+2.1 \\ 1.6 C-2 D-0.033 L-0.085 P-1.1 V-0.048 \phi-0.36 \\ 0.19 L-1.9 D-0.74 C-0.58 P-1.1 V-1.1 \phi+0.73 \\ 1.5 C+0.65 D+0.11 L-0.029 P+0.69 V+2.2 \phi-0.11 \\ 2.8 e-3 P-1.5-0.079 L-1.4 C-1.8 V-3.5 \phi+0.48 \\ 0.018 P-0.54 D-0.61 L-0.032 C+1.0 V-0.011 \phi-0.47 \\ 0.67 C+0.48 D+0.84 L-0.023 P-1.8 V+0.43 \phi+0.65 \\ 3.2 C+1.4 D-1 L-0.02 P-1.2 V+2.2 \phi+1.5\end{array}\right]$

Substituting and simplifying Eq. 32 in Eq. 31, Eq. 33 is obtained.

$\operatorname{tansig}(M)=\left[\begin{array}{c}\frac{2}{1+\exp (3 C+2.3 D-0.12 L+0.047 P+0.021 V-7.9 \phi-6)}-1 \\ \frac{2}{1+\exp (1.4 C-3.7 D-1.62-0.14 P-4.1 V+2.0 \phi+2.9)}-1 \\ \frac{2}{1+\exp (0.4 D-3.4 C+1.4 L+0.086 P+1.4 V+0.65 \phi-4.1)}-1 \\ \frac{2}{1+\exp (4.1 D-3.3 C+0.066 L+0.17 P+2.1 V+0.095 \phi+0.72)}-1 \\ \frac{2}{1+\exp (1.5 C+3.7 D-0.39 L+1.2 P+2.2 V+2.3 \phi-1.5)}-1 \\ \frac{2}{1+\exp (0.059 P-1.3 D-0.23 L-3.1 C-1.4 V-4.4 \phi+0.21)}-1 \\ \frac{2}{1+\exp (2.8 C+3.1 D+0.16 L-5.7 e-3 P+3.6 V+7 \phi-0.95)}-1 \\ \frac{2}{1+\exp (0.063 C+1.1 D+1.2 L-0.036 P-2.1 V+0.023 \phi+0.93)}-1 \\ \frac{2}{1+\exp (0.047 P-0.96 D-1.7 L-1.3 C+3.7 V-0.86 \phi-1.3)}-1 \\ \frac{2}{1+\exp (2 L-2.9 D-6.3 C+0.041 P+2.4 V-4.4 \phi-3)}-1\end{array}\right]$

The $Y$ output in Eq. 19 which is in this case the rolling resistance coefficient, $f_{r}$, becomes as shown in Eq. 34 after solving using Eq. 33 . 


$$
\begin{aligned}
f_{r}= & \frac{2.1}{\exp (2 L-2.9 D-6.3 C+0.041 P+2.4 V-4.4 \phi-3)+1} \\
& -\frac{0.63}{\exp (4.1 D-3.3 C+0.066 L+0.17 P+2.1 V+0.095 \phi+0.72)+1} \\
& -\frac{2.9}{\exp (0.063 C+1.1 D+1.2 L-0.036 P-2.1 V+0.023 \phi+0.93)+1} \\
& +\frac{2.4}{\exp (1.4 C-3.7 D-1.6 L-0.14 P-4.1 V+2 \phi+2.9)+1} \\
& -\frac{0.39}{\exp (1.5 C+3.7 D-0.39 L+1.2 P+2.2 V+2.3 \phi-1.5)+1} \\
& +\frac{1.1}{\exp (0.4 D-3.4 C+1.4 L+0.086 P+1.4 V+0.65 \phi-4.1)+1} \\
& +\frac{5.2}{\exp (0.059 P-1.3 D-0.23 L-3.1 C-1.4 V-4.4 \phi+0.21)+1} \\
& +\frac{4.1}{\exp (3 C+2.3 D-0.12 L+0.047 P+0.021 V-7.9 \phi-6)+1} \\
& +\frac{7.7}{\exp (2.8 C+3.1 D+0.16 L-5.7 e-3 P+3.6 V+7 \phi-0.95)+1} \\
& -\frac{1.9}{\exp (0.047 P-0.96 D-1.7 L-1.3 C+3.7 V-0.86 \phi-1.3)+1} \\
& -10.7
\end{aligned}
$$

However, Eq. 34 can not be simplified anymore and this is the final form that is adopted to compute the rolling resistance coefficient at different operating conditions.

\section{References}

1. Schmit LA, Farshi B (1973) Optimum laminate design for strength and stiffness. Int J Numer Methods Eng 7(4):519-536

2. Holland JH (1992) Genetic algorithms. Sci Am 267(1):66-73

3. Jiao L, Wang $L$ (2000) A novel genetic algorithm based on immunity. IEEE Trans Syst Man Cybern Part A Syst Hum 30(5):552-561

4. Abe A, Kamegawa T, Nakajima Y (2004) Optimization of construction of tire reinforcement by genetic algorithm. Optim Eng 5(1):77-92

5. Matuško J, Petrović l, Perić N (2008) Neural network based tire/ road friction force estimation. Eng Appl Artif Intell 21(3):442-456

6. Cho JR, Lee JH, Jeong KM, Kim KW (2012) Optimum design of run-flat tire insert rubber by genetic algorithm. Finite Elem Anal Des 52:60-70

7. Ćirović V, Aleksendrić D, Mladenović D (2012) Braking torque control using recurrent neural networks. Proc Inst Mech Eng Part D J Automob Eng 226(6):754-766

8. Zhu X, Dang L (2017) A 'smart tire' for estimating the tire force and the extents of the influences of specific factors. Proc Inst Mech Eng Part D J Automob Eng 231(13):1848-1857

9. McCulloch WS, Pitts W (1943) A logical calculus of the ideas immanent in nervous activity. Bull Math Biophys 5(4):115-133

10. El-Sayegh Z, El-Gindy M (2018) Modelling and prediction of tyre-snow interaction using finite element analysis-smoothed particle hydrodynamics techniques. Proc Inst Mech Eng Part D J Automob Eng 0954407018788997
11. El-Sayegh Z, El-Gindy M, Johansson I, Öijer F (2018) Improved tire-soil interaction model using FEA-SPH simulation. J Terramech 78C:53-62

12. Chae $S$ (2006) Nonlinear finite element modeling and analysis of a truck tire. PhD thesis, The Pennsylvania State University

13. PAM System International. Pam-crash user manual version 2014. ESI Group (2014)

14. El-Sayegh Z, El-Gindy M, Johansson I, Öijer F (2018) Off-road soft terrain modeling using smoothed particle hydrodynamics technique. In: ASME 2018 international design engineering technical conferences and computers and information in engineering conference. American Society of Mechanical Engineers, pp V003T01A030-V003T01A030

15. El-Sayegh Z, El-Gindy M (2017) Sensitivity analysis of truck tire hydroplaning speed using fea-sph model. Int J Veh Syst Modell Testing 12(1/2):143-161

16. El-Sayegh Z, El-Gindy M, Johansson I, Öijer F (2018) Modeling of interaction between tire and wet surface using finite element analysis and smoothed-particle hydrodynamics techniques. SAE Technical Paper, No 2018-01-1118

17. El-Sayegh Z, El-Gindy M, Johansson I, Öijer F (2018) Development of in-plane truck tire-flooded surface interaction models using FEA-SPH techniques. In: ASME 2018 international design engineering technical conferences and computers and information in engineering conference. American Society of Mechanical Engineers, pp V003T01A031-V003T01A031

18. El-Sayegh Z, El-Gindy M, Johansson I, Öijer F (2020) Modelling tire-moist terrain interaction using advanced computational techniques. J Terramech 91:23-30

Publisher's Note Springer Nature remains neutral with regard to jurisdictional claims in published maps and institutional affiliations. 